\title{
Propagation and feeding behavior of the mycetophagous nematode, Aphelenchus avenae, on four species of soil fungi
}

\author{
Nobuyoshi Ishibashi ${ }^{1}$, Sachiko Takayama ${ }^{1}$ and Eizo Kondo ${ }^{1,2}$
}

\begin{abstract}
Three isolates of the mycetophagous nematode Aphelenchus avenae collected in Kyushu, Japan, were investigated at $25^{\circ} \mathrm{C}$ for their propagation and feeding behavior on the hyphae of Rhizoctonia solani (Rs), Botrytis cinerea (Bc), Fusarium oxysporum (Fo), and Verticillium dahliae $(\mathrm{Vd})$. There were large differences in the population growth between the nematode isolates. On 30 days after inoculation with three individuals of 4 th-stage juveniles onto the fungi growing on the $1 / 5$ strength PDA medium in a Petri dish $(9-\mathrm{cm}$ diam.), the nematode population size was in the range of 21,000 - 63,000 for Bc and Rs, and 1,000 - 10,000 for $\mathrm{Vd}$ and Fo. Taking the hyphal diameter of Rs for 1.0, the ratio was almost the same, 0.2 , and 0.4 for $\mathrm{Bc}, \mathrm{Vd}$, and $\mathrm{Fo}$, respectively. Video-assisted analyses of nematode feeding behavior showed that the nematodes were more preferably attracted to Fo than to the other three fungus species. The frequencies of stylet thrusting per $10 \mathrm{~min}$ were 5 - 12 times for Rs and $\mathrm{Bc}$, and 20 - 106 times for Fo or $\mathrm{Vd}$. The duration of a single stylet stabbing was 4 - 9 sec for Rs and Bc, while it was 1 - 2 sec for Fo and Vd. The pulsation frequencies of median valve per a single feeding were 5 - 10 pulse for Rs and $\mathrm{Bc}$, and $0.5-1.0$ pulse for Fo and Vd. The lower nematode reproduction on Fo and Vd was possibly due to the thinner width of these hyphae than the length of nematode stylet (ca. $8 \mu \mathrm{m}$ ). There is no extracorporeal digestion in these three nematode isolates tested. Jpn. J. Nematol. 35(1), 13 - 19 (2005).

Key words: Aphelenchus avenae, mycetophagous nematode, feeding behavior, propagation.
\end{abstract}

\section{INTRODUCTION}

Aphelenchus avenae is a ubiquitous fungivorous nematode which feeds on hyphae of more than 90 species of soil fungi (Ishibashi, unpublished; Townshend, 1964). Since most of these fungi are phytopathogenic, many investigations have been conducted toward the use of the nematode as a biological agent of soil borne fungal diseases (Mankau and Mankau, 1963; Gupta, 1986; Barker, 1964; Barnes et al., 1981; Choo and Estey, 1985; Ishibashi, 1990). In order to make $A$. avenae an efficient bio-control agent of fungal

\footnotetext{
${ }^{1}$ Saga University, Honjo 1, Saga 840 - 8502, Japan.

${ }^{2}$ Correspondence author; kondoe@cc.saga-u.ac.jp
}

disease of plants, an establishment of nematode mass culture is prerequisite. However, population growth of the nematode under culture condition is unstable depending on the nematode isolates collected from various localities and on the nematode preference toward the fungi used as food (Ali et al., 1999; Choi and Ishibashi, 1989). In general, Botrytis cinerea and Rhizoctonia solani are favorable for $A$. avenae (Nickle and McIntosh, 1968; Ikonen, 2001; Ishibashi et al., 2000; Choi and Ishibashi, 1989), while Fusarium, Verticillium, and Pythium are meager for the nematode (Choi and Ishibashi, 1989). The different nematode propagation is possibly due to the toxic substances produced by the host fungi (Mankau, 1969; Brian, 1951) and to the morpho- 
logical barriers of conidiophores and/or sporulation (Ruess et al., 2000).

As to tylenchid mycetophagous nematodes, Doncaster (1966) reported in detail a feeding habit on $B$. cinerea and an extracorporeal digestion by dorsal gland secretions of Ditylenchus destructor and D. myceliophagous. For aphelenchid mycetophagous nematodes, Fisher and Evans (1967) demonstrated no injection of esophageal secretions into host cells; $A$. avenae ingested contents of host fungi without an extracorporeal digestion. Although these investigations were mostly focusing upon a combination of a given host fungus and a single nematode isolate, further studies on various nematode isolates - host fungi combinations have been limited so far.

The present study was conducted to compare the suitability of four host fungus species for the reproduction and feeding behavior of three isolates of $A$. avenae collected from Kyushu, Japan.

\section{MATERIALS AND METHODS}

Nematodes:

Three isolates of Aphelenchus avenae used in the present study were collected from Nagasaki, Saga, and Ohita prefectures which locate in northern Kyushu, Japan. Otherwise particularly mentioned, all of these isolates were cultured at $25^{\circ} \mathrm{C}$ on fungal mat of Botryitis cinerea growing on $1 / 5$ strength medium of PDA (potato $40 \mathrm{~g}$, dextrose $4 \mathrm{~g}$, agar $15 \mathrm{~g}$ ) in a Petri dish (9 - cm diam.). The 4th stage juveniles and young adults were washed off from the side wall and the lid of Petri dish and kept at $5{ }^{\circ} \mathrm{C}$ in distilled water for 2 to 3 days until use as nematode inocula.

Host fungi:

Rhizoctonia solani AG-4, Botrytis cinerea, Fusarium oxysporum f. sp. melonis, and Verticillium dahliae were used as host fungi to study propagation and host preference of $A$. avenae. Prior to use these fungi as hosts, they were cultured on $1 / 5$ strength PDA at $20-25^{\circ} \mathrm{C}$ and kept at $5-10^{\circ} \mathrm{C}$ until use as fungal inocula.

Propagation of nematodes on fungus hyphae:

The fungal mats (10 - mm diam. disk) of $F$. oxysporum, $V$. dahliae, B. cinerea, and $R$. solani growing on $1 / 5$ strength PDA media in Petri dish $(9-\mathrm{cm}$ diam.) were inoculated with one of the three isolates of $A$. avenae at the density of three 4th stage juveniles per dish. Thirty days after inoculation, nematodes were recovered by Baermann funnel technique and counted. The experiment was performed twice with five replicates.

Observation of feeding behavior:

The square fungal mat $(18 \times 18 \mathrm{~mm})$ of host fungi was cut out from marginal portion of actively growing fungal hyphae, transferred onto a concaved slide glass, and inoculated with one of the three nematode isolates at the density of 20 4th stage juveniles and/or young female adults per slide glass. To allow evaporate distilled water used to suspend nematode inocula (10 $\mu 1)$ and to clearly observe nematode behavior, a cover slip was applied over the slide glass about two hours after nematode inoculation.

The movement of stylet and median valve of the nematode was recorded under a microscope at the magnification of 600 using a video recorder equipped with a video-timer (Hitachi VL-S100). Recording was made for $10 \mathrm{~min}$ each for six individuals of the three nematode isolates inoculated onto one of four fungus species tested.

The recorded image of nematode feeding behavior was play-backed at the $1 / 5$ speed of the natural speed to make easily analyze behavioral characteristics of the three nematode isolates on the four fungus species. The criteria employed to make comparative analysis were: 1 ) the time from the onset of stylet stabbing into fungal 
hyphae to the stopping of median valve pulsation, 2) the duration of pulsation of valve for one time feeding, 3) the frequency of pulsation per one feeding, and 4) the frequency of feeding per $10 \mathrm{~min}$.

Measurement of hyphae:

Average width and density of hyphae of the four fungus species were calculated by measuring hyphae at ten randomly selected portions of the recorded video images. The hyphal width of the respective fungus examined was expressed as the ratio against the hyphal width of $R$. solani (=1.0). The hyphal density was recorded as numbers of hyphae in a given area on the video screen. Measurements were made for the hyphae taken from five Petri dishes.

Data analysis:

The numerical data obtained were analyzed using a T-test $(\alpha=0.05)$.

\section{RESULTS}

Propagation of $A$ avenae on four fungus species:

The population growth of $A$. avenae on the four tested fungus species is shown in Table 1. The propagation of all the three nematode isolates was greater in the order of $R$. solani, $B$. cinerea, F. oxysporum, and $V$. dahliae, excepting Ohita isolate whose propagation on the former two fungi was not significantly different from each other. On R. solani, Nagasaki and Saga isolates propagated most profoundly and reached to the population size of ca. 60,000 on 30 days after inoculation with three individuals of $A$. avenae. The propagation of the three isolates on $B$. cinerea was superior to the propagation on $F$. oxysporum and $V$. dahliae. The latter two fungi were poor hosts for $A$. avenae whose population was ca. 10,000 or less at the end of propagation test period.

Width and density of fungal hyphae:

The hyphal width of $F$. oxysporum, and $V$. dahliae was far more narrower than that of $R$. solani and B. cinerea (Fig. 1). Compared to the body width and stylet length (ca. $8 \mu \mathrm{m}$ ) of $A$. avenae, the former two hyphae seemed to be too small for the nematode to take the hyphal contents sufficiently. The relative thickness of hyphae was greater in the order of $R$. solan $i=B$. cinerea, F. oxysporum, and $V$. dahliae. The hyphae of the latter two were significantly thinner than those of the former two (Table 2). Contrarily, the densities of fungal hyphae of $F$. oxysporum and $V$. dahliae were significantly greater than that of $R$. solani and B. cinerea.

Feeding behaviour of $A$. avenae on four fungus species:

Video-assisted observation revealed that $A$. avenae began to probe the wall of fungal hyphae

Table 1. Propagation of three isolates of Aphelenchus avenae on four species of fungi.

\begin{tabular}{lccc}
\hline \multirow{2}{*}{\multicolumn{1}{c}{ Host fungi }} & \multicolumn{3}{c}{ Number of nematodes produced per Petri dish $\left(\times 10^{4}\right)$} \\
\cline { 2 - 4 } & Nagasaki & Ohita & Saga \\
\hline Rhizoctonia solani & $6.3 \pm 0.7 \mathrm{a}$ & $2.1 \pm 0.2 \mathrm{c}$ & $5.8 \pm 0.8 \mathrm{a}$ \\
Botrytis cinerea & $3.8 \pm 0.5 \mathrm{~b}$ & $2.3 \pm 0.5 \mathrm{c}$ & $2.3 \pm 0.3 \mathrm{c}$ \\
Fusarium oxysporum & $1.0 \pm 0.2 \mathrm{~d}$ & $0.6 \pm 0.1 \mathrm{e}$ & $0.2 \pm 0.1 \mathrm{e}$ \\
Verticillium dahliae & $0.4 \pm 0.1 \mathrm{f}$ & $0.1 \pm 0.1 \mathrm{f}$ & $0.1 \pm 0.1 \mathrm{f}$ \\
\hline
\end{tabular}

Nematode production was examined 30 days after inoculation of three 4 th stage juveniles of $A$. avenae on the fungal mat of tested fungi growing at $25^{\circ} \mathrm{C}$ on the $1 / 5$ strength PDA medium in Petri dish (9- $\mathrm{cm}$ diam.). Data are the mean. Numerals (Mean \pm S.D.) with the same alphabetical letters are not significantly different at the level $0.05(\phi \leqq 0.05)$. 


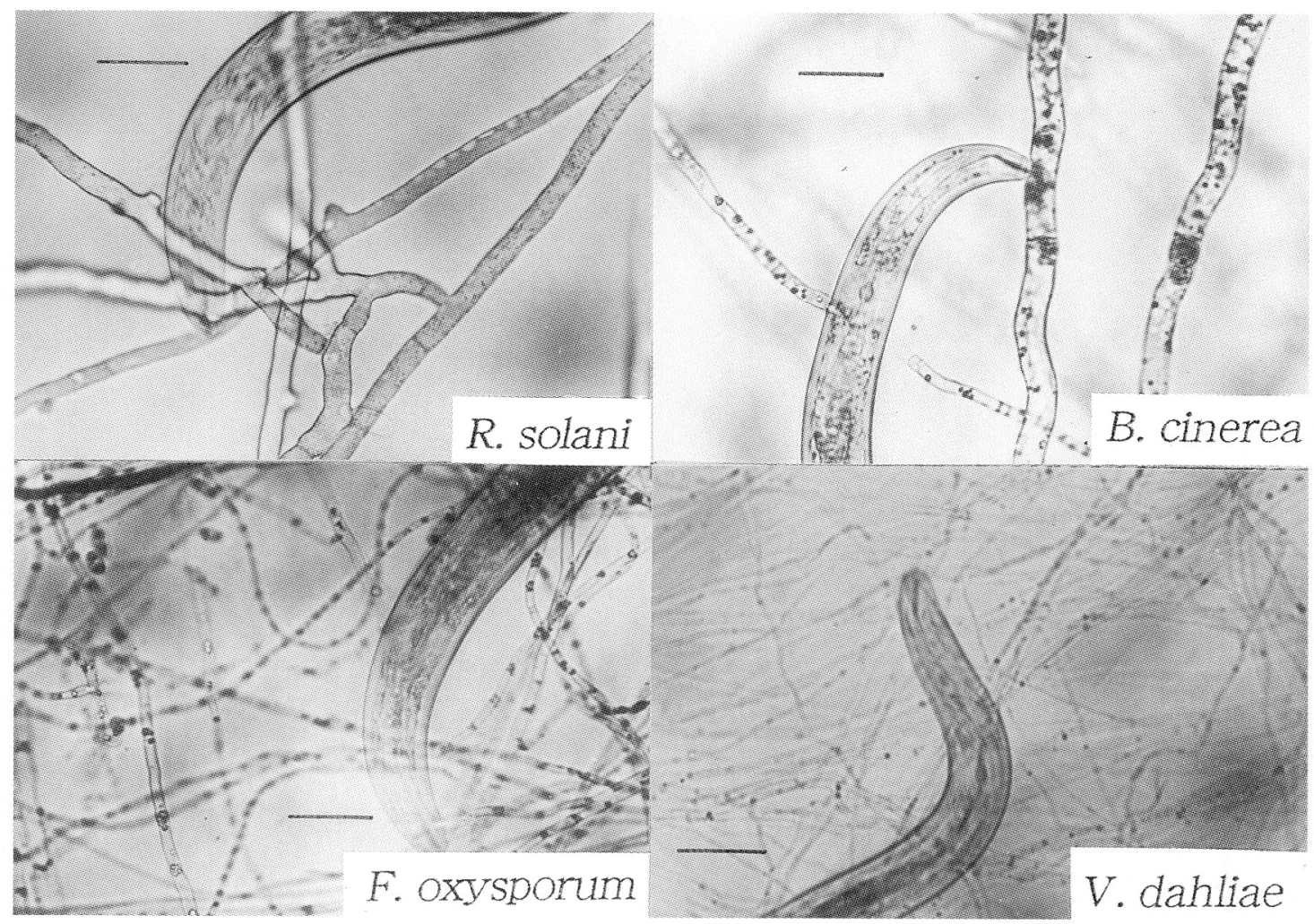

Fig. 1. Aphelenchus avenae feeding on the hyphae of Rhizoctonia solani, Botrytis cinerea, Fusarium oxysporum f. sp. melonis, and Verticillum dahliae. Bars show $10 \mu \mathrm{m}$.

Table 2. Width ratio and density of hyphae of tested four fungi.

\begin{tabular}{lcc} 
& \multicolumn{2}{c}{ Mean \pm S.D. } \\
\hline \multicolumn{1}{c}{ Host fungi } & \multicolumn{2}{c}{ Fungal hyphae } \\
& Width $^{1}$ & Density $^{2}$ \\
\hline Rhizoctonia solani & $1.0 \pm 0.2 \mathrm{a}^{3}$ & $9.0 \pm 2.0 \mathrm{a}$ \\
Botrytis cinerea & $0.9 \pm 0.1 \mathrm{a}$ & $8.0 \pm 1.5 \mathrm{a}$ \\
Fusarium oxysporum & $0.4 \pm 0.1 \mathrm{~b}$ & $46.3 \pm 8.2 \mathrm{~b}$ \\
Verticillium dahliae & $0.2 \pm 0.1 \mathrm{c}$ & $38.0 \pm 8.3 \mathrm{~b}$ \\
\hline
\end{tabular}

${ }^{1}$ The hyphal width of the fungi tested is expressed as the ratio against the hyphal width of $R$. solani $(=1.0)$.

${ }^{2}$ The hyphal density is the average number of hyphae at ten randomly selected area of fungal mat on the video screen.

${ }^{3}$ Numerals with the same alphabetical letters are not significantly different at $\alpha=0.05$ level. with stylet upon the contact of nematode head with the wall of hyphae. Immediately after stylet stabbing, pulsation of median valve of metacorpus was followed. This valval pulsation was terminated when the cell content of hyphae of host fungi was ingested, and thereafter the nematode left the exhausted fungal hyphae.

The pulsation time of all the three nematode isolates was longer in the order of $R$. solani, $B$. cinerea, F. oxysporum, and $V$. dahliae (Table 3). Although there was no statistical difference, the three nematode isolates tended to pulsate longer on $R$. solani than on $B$. cinerea. On these two fungi, Saga strain pulsated longer than Nagasaki and Ohita isolates. The pulsation time on $F$. oxysporum and $V$. dahliae was significantly shorter than on $R$. solani and $B$. cinerea; it was $1.3-1.5 \mathrm{sec}$ on $F$. oxysporum and $0.5-0.8$ sec on $V$. dahliae, while the duration for $R$. solani 
Table 3. Pulsation time (sec) of median valve of $A$. avenae for one feeding.

\begin{tabular}{lccc}
\hline \multirow{2}{*}{ Host fungi } & \multicolumn{3}{c}{ Isolates of A. avenae } \\
\cline { 2 - 4 } & Nagasaki & Ohita & Saga \\
\hline Rhizoctonia solani & $6.5 \pm 1.5 \mathrm{a}$ & $6.5 \pm 2.0 \mathrm{a}$ & $9.3 \pm 2.0 \mathrm{a}$ \\
Botrytis cinerea & $3.5 \pm 1.0 \mathrm{a}$ & $4.8 \pm 2.0 \mathrm{a}$ & $8.5 \pm 5.0 \mathrm{a}$ \\
Fusarium oxysporum & $1.5 \pm 0.2 \mathrm{c}$ & $1.3 \pm 0.2 \mathrm{c}$ & $1.5 \pm 0.2 \mathrm{c}$ \\
Verticillium dahliae & $0.5 \pm 0.1 \mathrm{~d}$ & $0.8 \pm 0.1 \mathrm{~d}$ & $0.8 \pm 0.1 \mathrm{~d}$ \\
\hline
\end{tabular}

Numerals (Mean \pm S.D.) with the same alphabetical letters are not significantly different at $\alpha=0.05$.

Table 4. Feeding frequencies of Aphelenchus avenae for $10 \mathrm{~min}$ on tested four fungi .

\begin{tabular}{lccc}
\hline \multirow{2}{*}{\multicolumn{1}{c}{ Host fungi }} & \multicolumn{3}{c}{ Isolates of A. avenae } \\
\cline { 2 - 4 } & Nagasaki & Ohita & Saga \\
\hline Rhizoctonia solani & $5.0 \pm 1.0 \mathrm{ab}$ & $12.0 \pm 4.3 \mathrm{~b}$ & $5.0 \pm 1.0 \mathrm{ab}$ \\
Botrytis cinerea & $12.0 \pm 2.0 \mathrm{~b}$ & $6.0 \pm 5.4 \mathrm{ab}$ & $6.0 \pm 2.0 \mathrm{ab}$ \\
Fusarium oxysporum & $48.0 \pm 9.8 \mathrm{c}$ & $42.3 \pm 4.0 \mathrm{c}$ & $20.2 \pm 7.5 \mathrm{~d}$ \\
Verticillium dahliae & $106.0 \pm 30.5 \mathrm{e}$ & $53.5 \pm 15.3 \mathrm{c}$ & $43.0 \pm 13.8 \mathrm{c}$ \\
\hline
\end{tabular}

Numerals with the same alphabetical letters are not significantly different at $\alpha=0.05$ level

and B. cinerea was 4 - 9 sec.

The feeding frequency of the three nematode isolates was the greatest on $V$. dahliae, followed by $F$. oxysporum, B. cinerea, and $R$. solani (Table 4).

On $V$. dahliae the frequency per 10 min was ca. 43 times for Saga isolate, ca. 54 for Ohita isolate, and ca. 106 for Nagasaki isolate. The frequency of these three isolates on $R$. solani was ca. 5.0, 12.0, and 5.0 times, respectively. Excepting Ohita isolate on $R$. solani, the frequency of Nagasaki isolate was greater than the other two isolates on B. cinerea, F. oxysporum, and $V$.dahliae.

\section{DISCUSSION}

The fungivorous nematode Aphelenchus avenae has a high potential to propagate itself by feeding upon various kinds of fungi in cultivated and non-cultivated soil, thus making it widely distribute worldwide. However the propagation rate of this nematode is considerably different depending on the kinds of host fungi (Ali et al., 1999).
In the present study, propagation of the three isolates of $A$. avenae was significantly different due to the four fungus species used as nematode food. Generally the fungi $F$. oxysporum and $V$. dahliae were not suitable for all of the tested nematode isolates, although these two fungus species did not show any repulsive response against the nematode and attracted more nematodes than the suitable host fungi $R$. solani and B. cinerea (data not shown). We did not study nematode attraction substances emanating from tested fungi, but the nematode attraction by host fungi may not correlate with their suitability as food for the nematode, as was suggested by Ruess et al. (2000).

Regarding negative factors for nematode propagation, production of such toxic metabolites as antibiotics by fungi and such morphological barriers as formation of conidiophores and/or sporulation of fungi had been proposed (Ruess et al., 2000). Considering toxic properties purportedly produced by fungi (Briani, 1951; Mankau, 1969), chemical factors produced by fungi are to be comparatively investigated in 
addition to the study on mechanical barrier against nematode feeding.

Depending on the width and density of hyphae, the four fungus species used were divided into two groups: the fungi with the wider hyphae and lower density ( $R$. solani and $B$. cinerea) and those with the narrower hyphae and higher density ( $F$. oxysporum and $V$. dahliae). Namely the nematode production was higher on the former two fungi and lower on the latter two, being indicative of no positive relation of nematode propagation to hyphal density and of positive relation to hyphal width. Considering a feeding behavior and the depth of stylet thrusting into fungal cells, the lower nematode production rate on $F$. oxysporum and $V$. dahliae may be caused, at least partly, by the thinner width of these hyphae than the nematode stylet length, resulting in the food uptake insufficient to support nematode growth and propagation.

In the present study we showed that the propagation of the three isolates of $A$. avenae was considerably different due to the species of fungi used as food. In order to understand more deeply an intra-strain difference of $A$. avenae and an inter-species difference of host fungi, chemical and physical factors involved in the pre-feeding and post-feeding phase remain to be further analyzed.

\section{LITERATURE CITED}

Ali, M. D., Amin, B., Adachi, T., and Ishibashi, N. (1999) Host and temperature preference, male occurrence and morphometrics of fungivorous nematode, Aphelenchus avenae isolates from Japan. Japanese Journal of Nematology 29, 7-17.

Barker, K. P. (1964) On the disease reduction and production of the nematode Aphelechus avenae on isolates of Rhizoctonia solani. Plant Disease Reporter 48, 428-432.
Barnes, G. L., Russel, C. C., Foster, W. D., and Mcnew, R. W. (1981) Aphelenchus avenae, a potential biological control for root rot fungi. Plant Disease 65, 423-424.

Brian, P. W. (1951) Antibiotics produced by fungi. The Botanical Review 6, 357-430.

Choi, D. R. and Ishibashi, N. (1989) Propagation of five Aphelenchus avenae isolates on six species of fungi and five substrates. Japanese Journal of Nematology 19, 13-17.

Choo, P. H. and Estey, H. (1985) Control of the damping-off disease of pea by Aphelenchus avenae. Indian Journal of Nematology 15, 14.

Doncaster, C. C. (1966) Nematode feeding mechanisms. 2. Observations on Ditylenchus destructor and D. myceliophagus feeding on Botrytis cinerea. Nematologica 12, 417-427.

Fisher, J. M. and A. A. F. Evans (1967) Penetration and feeding by Aphelenchus avenae. Nematologica 13, 425-428.

Gupta, M. C. (1986) Biological control of Fusarium moniliformae Sheldon and Pythium butleri Subramanium by Aphelenchus avenae Bastian, 1985 in chitin and cellulose amended soils. Soil Biology and Biochemistry 18, 328-329.

Ikonen, Eeva K. (2001) Population growth of two aphelenchid nematodes with six different fungi as a food source. Nematology 3, 9-15.

Ishiabshi, N. (1990) Development of biological integrated control of agricultural pests by beneficial nematodes. In: Tech. Bull. (Grantin-Aid for Developmental Scientific Research, Ministry of Education, Culture and Science, Japan. Grant No. 6286006, Saga University, Saga 840-8502, Japan) pp.128131. Pp. 159

Ishibashi, N., Ali, M. R., and Saramoto, M. (2000) Mass-production of fungivorous nematode, Aphelenchus avenae Bastian 1865, on industrial vegetable/animal wastes. Japanese 
Journal of Nematology 30, 8-17.

Mankau, R. (1969) Toxicity of culture filtrates of Aspergillus niger to the mycophagous nematode, Aphelenchus avenae. Phytopathology 59, 13.

Mankau, R. and Mankau, S. K. (1963) The role of mycophagous nematodes in soil. I. The relationships of Aphelenchus avenae to phytopathogenic soil fungi. In: Soil organisms (eds. Doekson, J. \& van der Drift, J.), Amsterdam, The Netherlands, NorthHolland Publishing Company, 271-280.

Nickle, W. R. and McIntosh, P. (1968) Studies on the feeding and reproduction of seven mycophagous nematodes on Rhizoctonia,
Fusarium, and Verticillium. Nematologica 14, 11-12.

Ruess, L., Garcia Zapata, E. J., and Dighton, J. (2000) Food preferences of a fungal feeding Aphelenchoides species. Nematology 2, 223230.

Townshend, J. L. (1964) Fungus hosts of Aphelenchus avenae Bastian, 1865, and Bursaphelenchus fungivorous Franklin and Hooper, 1962 and their attractiveness to these nematode species. Canadian Journal of Microbiology 10, 727-737.

Received October 29, 2004. 